\title{
Die Anfänge jüdischer Belletristik im 19. Jahrhundert
}

Mit theoretischen, oft allzu schulmeisterlichen Schriften ließ sich eine breite Öffentlichkeit nicht erreichen, geschweige denn die Herzen gewinnen. Dazu eignete sich indes jenes Medium, das eine besondere Bedrohung für den bewussten Verbleib in der jüdischen Gemeinschaft bei sich allmählich auflösender Pflichtschuld darstellte: die schöne Literatur. »Vor Einem hüte Deinen Sohn, Vater, Deine Tochter, Mutter! das ist vor dem Romanlesen!« wetterte Ludwig Philippson 1855 auf der Titelseite des Jüdischen Volksblatts, um den verderblichen Einfluss der sog. »Lesesucht« in drastischen Bildern zu schildern:

Das häufige Lesen von Romanen ist das Gift der Jugend, Gift für den Verstand, Gift für das Herz, Gift für die Bildung, Gift für das wirkliche Leben! Das Romanlesen ist dem Essen des Opiums, ist dem Rausche durch Branntwein gleich. Die Leihbibliotheken sind die offenen Buden dieser Vergiftung - legen ja doch schon die Schulen ähnliche Boutiken an! Giebt man doch schon recht wohlfeile Ausgaben, damit ein jeder sich selbst ein Magazin des entnervenden, betäubenden Stoffes anlegen könne. Ja, die Romane wie das Opium, bringen Rückgratsverkrümmungen des Geistes hervor. Erst Mährchen, dann Erzählungen, dann Romane - das ist der Weg! ${ }^{1}$

Philippsons Anwürfe gegen die Romane seiner Zeit waren keineswegs neu. Seit Anfang des 19. Jahrhunderts ein effizienteres Distributionssystem (Stichwort »Leihbibliotheken«) belletristische Schriften zugänglicher gemacht hatte und späterhin Feuilletonromane und preiswerte Ausgaben die Leserinnen und Leser mit Stoff versorgten, kamen in immer rasanterer Folge neue Romane auf den Markt, die von der zeitgenössischen Literaturkritik als »Trivialliteratur « angeprangert wurden. Die Handlung schematisch, die Figuren typenhaft, boten die zahllosen historischen und sentimentalen Liebesromane, Schauer- und Abenteuergeschichten, etc. leichte Unterhaltung und Ablenkung, die, wie ernste Gemüter warnten, Gefahr lief, ihre Leser und vor allem Leserinnen der Wirklichkeit zu entrücken. »Weltflucht« und intellektuelle Abstumpfung gehörten zu den immer wieder vorgebrachten moralischen Bedenken, während sich die ästhetischen »Vergehen « der Romanschreiber - anachronistisch gesprochen - unter der Kategorie »Kitsch« subsumieren ließen.

1 [Ludwig Philippson:] Vor Einem hüte Deinen Sohn, Vater, Deine Tochter, Mutter! In: Jüdisches Volksblatt 2 (1855), Nr 26, S. 101-102. - Das Jüdische Volksblatt (1854-1866) erschien in Leipzig, Herausgeber war Philippson selbst.

2 Open Access. (C) 2020 Almut Laufer, publiziert von De Gruyter. (œ) Br der Creative Commons Attribution 4.0 Lizenz.

https://doi.org/10.1515/9783110674255-007 
Als Philippson sein ingrimmiges Pamphlet gegen die zeitgenössische Unterhaltungsliteratur vom Stapel ließ, ging die von ihm gegründete, herausgegebene und auch redigierte Allgemeine Zeitung des Judenthums bereits in ihr 19. Jahr. Seit ihrem ersten Erscheinen 1837 hatte sie - zweifelsohne das wichtigste Organ des liberalen Judentums - Novellen in Fortsetzung, Lebensbilder, Skizzen und Erzählungen aller Art als fixen Bestandteil ihres Feuilletons geführt. ${ }^{2}$ Kritisch betrachtet wurden diese literarischen Texte kaum den Ansprüchen hoher Literatur gerecht, sondern ähnelten in vielerlei Hinsicht jenen Romanen, die Philippson so abstoßend fand. Literarische Verfahren, Handlungsaufbau und die Idiomatik eines gattungsspezifischen Stils ließen durchblicken, von welchem Modell man sich hatte inspirieren lassen. Zweifellos ging es Philippson und seinen Mitstreitern an der literarischen Front darum, ihre Erzählungen ansprechend und mitreißend, kurz: volksnah zu gestalten. Schließlich schrieb man nicht für die intellektuelle Elite, sondern für einen sich formierenden, bildungshungrigen Mittelstand, dem die Lektüre zeitgenössischer Schriften in Landessprache kultureller Fixpunkt war. Worin bestand nun aber der Unterschied zu den verfemten Romanen?

Zunächst ganz augenfällig in der Wahl des Milieus und des (historischen) Stoffes. Bei den von Philippson veröffentlichten Erzählungen handelte es sich um Adaptionen bekannten Novellen- und Romanguts und dessen Verlagerung in die Sphäre jüdischer Lebenswelt und Geschichtsauffassung. Als mithin konfessionelle Literatur konnten sich die Texte einer klaren Positionierung in weltanschaulichen und ethischen Fragen nicht entziehen, die sie letztlich ja auch bezweckten, nämlich: das Judentum in Zeiten des Umbruchs ideologisch auf eine neue Grundlage zu stellen. Nicht umsonst lautete der Untertitel des Jüdischen Volksblatts: »Zur Belehrung und Unterhaltung auf jüdischem Gebiete« [Hervorhebung A. L.]. Philippson war nicht allein Publizist und Romanautor, er war Rabbiner und seiner liberalen Amtsauffassung nach dafür verantwortlich, die jüdische Religion unbeschadet, wenngleich »geläutert«, durch die Zeit zu tragen. Sein Eintreten für die Belange einer neuen israelitischen Literatur stand also im Dienst einer Sache, die mehr mit Ideal und Moral als mit ästhetisch-literaturhistorischen Fragen zu tun hatte. Aus Philippsons Sicht fielen die literarischen Beiträge der Allgemeinen Zeitung des Judent(h)ums unter »ernste, belehrende Lectüre «. ${ }^{3}$

2 Zur Geschichte der AZJ s. Hans Otto Horch: Auf der Suche nach der jüdischen Erzählliteratur. Die Literaturkritik der »Allgemeinen Zeitung des Judentums« (1837-1922). Frankfurt a. M.: P. Lang 1985 (Literaturhistorische Untersuchungen; 1), S. $13 \mathrm{ff.}$

3 [Philippson,] Vor Einem hüte Deinen Sohn, Vater, Deine Tochter, Mutter! (wie Anm. 1), S. 101. 
Die verschlungenen Intrigen und Abenteuer der Protagonisten, all die heimlich zugeworfenen Blicke zweier Liebender und schwelgerischen Landschaftsbeschreibungen waren also zweckgebunden. Indem sich der Leser mit den allzeit vorbildlich agierenden jüdischen Helden identifizierte, sollte er sein eigenes Judentum und seine Stellung in der Gesellschaft reflektieren und stolz und zuversichtlich in seiner Treue zur angestammten Glaubensgemeinschaft bestärkt werden. Eine neue jüdische Volks- oder Populärliteratur in deutscher Sprache war dazu berufen, eine verhängnisvolle Leerstelle auszufüllen, bevor die zeitgenössische Unterhaltungs- und Trivialliteratur mit ihren wenig tugendhaften und noch weniger jüdischen Protagonisten diese besetzen - und die Herzen abspenstig machen konnte. ${ }^{4}$

Die Aufgabe der Unterhaltung, Belehrung und Erbauung hatte über Jahrhunderte - um die erwähnte Leerstelle zu benennen - die jiddische Literatur innegehabt. »Auch wir hatten bis vor ungefähr zwei Dezennien unsre Volksbücher. Das Simchath-nephesch, der Weiberspiegel, das Samuel-Buch, Ritter Widwilt u. a. m. waren zu ihrer Zeit sehr brauchbare Schriften. Sie unterhielten, belehrten und erbauten das Volk zugleich und entsprachen somit ihrem Zwecke«, schreibt Simon Krämer 1839 in der Vorrede zu seiner ersten Volks- und Jugendschrift. Und weiter heißt es:

Eine andere Zeit ist an uns heraufgezogen, neue Verhältnisse haben sich gestaltet, neue Interessen sich geltend gemacht, die Volksbildung ist im Zunehmen. Grund genug, jene Werke nicht mehr genügend zu finden, wenn nicht die schiefen Weltansichten ihrer Verfasser, ins Besondere aber ihr korupter, unästhetischer Styl sie aller Brauchbarkeit für unsre Zeit beraubt und bereits zum Gegenstande des Spottes bei denen gemacht hätte, welchen sie nützen sollen. ${ }^{5}$

4 Erst die technischen Neuerungen in der zweiten Hälfte des 19. Jahrhunderts sorgten für eine weite Verbreitung von erschwinglichem Lesestoff. Zumindest Simon Krämer gesteht, dass die "schalen Romane « und »verflachenden Geistesprodukte der sogenannten modernen Literatur, zu welchen viele ihre Zuflucht nehmen, [...] Gottlob! dem größten Theil des Volkes unzugänglich«sind (Simon Krämer: אלה תולדות משפחת יחזקאל האך oder Die Schicksale der Familie Hoch. Ein Lesebuch zunächst für das jüdische Volk und seine reifere Jugend. Dinkelsbühl: Friedrich Walthr 1839, S. IV). - Für einen kurzen Überblick über die Verfügbarkeit von Belletristik in Deutschland s. The German Bestseller in the Late Nineteenth Century. Ed. by Charlotte Woodford and Benedict Schofield. Rochester. New York: Camden House 2012 (Studies in German Literature, Linguistics, and Culture), Introduction.

5 Krämer, Die Schicksale der Familie Hoch (wie Anm. 4), S. III-IV. - Das Sefer simkhat ha-nefesh (Frankfurt 1707, Fürth 1727) von Elchanan Hendel ben Benjamin Wolf Kirchhan war eines der bekanntesten Mussar-Bücher (Jean Baumgarten: Introduction to Old Yiddish Literature. Ed. and transl. by Jerold C. Frakes. Oxford: Oxford University Press 2005, S. 209f.); das Schmuel-bukh 
In seiner Aufzählung von Mussar-Büchern, legendenhaften Nacherzählungen biblischer Stoffe und höfischen Ritterepen des 16., 17. und frühen 18. Jahrhunderts beanstandet Krämer, seines Zeichens Dorfschullehrer in Mittelfranken, ${ }^{6}$ die Unzulänglichkeiten einer in jeder Hinsicht »veralteten « Literatur. Die Verwendung des Jiddischen oder Judendeutschen entlockte all jenen, denen die deutsche Standardsprache bereits vertrauter war als das jüdische Idiom, ein abfälliges Urteil ganz nach dem Muster eines aufgeklärten Sprachpurismus: Das Dargebotene wirkte archaisch, verderbt und primitiv. Und weltanschaulich hinkte es erst recht: Klar wie die Grenzen dieser literarischen Gattungen waren die sozialen einer »altständischen, hierarchisch-korporativen Ordnung « ${ }^{7}$ und Gruppenbindung, welcher sich der Einzelne bedingungslos unterzuordnen hatte. In Konflikt dazu geriet er erst, als religiöse Zugehörigkeit, bürgerlicher Stand, Bildungsgrad und Vermögensverhältnisse sich als unabhängige Parameter der eigenen Standortbestimmung innerhalb des gesellschaftlichen Ganzen voneinander zu lösen begannen. Roman und Novelle waren mit ihrer strukturellen Offenheit für konfligierende Lebensentwürfe, Selbstverwirklichung und Scheitern ihrer Protagonisten am Puls der Zeit - einer Zeit, in der sich Religiosität von Religion abzulösen begann.

Was die neuen Volksschriften bezwecken sollten, war die »Beförderung ächter Humanität«, die »Bildung des Geistes und Herzens «, ${ }^{8}$ wie Abraham Kohn in seinem ebenfalls 1839 erschienenen Aufsatz schreibt. Mit Stolz auf die unge-

(Augsburg 1544) eine Nacherzählung des biblischen Stoffes nach dem Vorbild des höfischen Heldenepos (ebd., S. 143) und Widuwilt (auch unter Kinig Artis houf bekannt) ein Artusroman (ebd., S. 158ff; s. auch Achim Jaeger: Ein jüdischer Artusritter. Studien zum jüdisch-deutschen »Widuwilt« [»Artushof«] und zum »Wigalois« des Wirnt von Gravenberc. Tübingen: Niemeyer 2000 [Conditio Judaica. Studien und Quellen zur deutsch-jüdischen Literatur- und Kulturgeschichte; 32]). - Das Simkhat ha-nefesh erwähnt auch Julius Dalberg in seiner Volkskunde der Hessen-Kasseler Juden (Kassel: Israelitische Gemeinde Kassel 1932, S. 126).

6 Simon Krämer, geb. 1808 in Schnaittach und gest. 1887 in Nürnberg, hatte das Lehrerbildungsseminar in Altdorf besucht und war danach jahrzehntelang als Religions- und Elementarlehrer in Mittelfranken tätig. Er vertrat reformjüdische Positionen und betätigte sich sowohl journalistisch als auch literarisch. Zu Krämer s. Falk Wiesemann: Simon Krämer (1808-1887), ein jüdischer Dorfschullehrer in Mittelfranken. In: Geschichte und Kultur der Juden in Bayern. Lebensläufe. Hg. von Manfred Treml und Wolf Weigand unter Mitarbeit von Evamaria Brockhoff. München: K. G. Saur 1988, S. 121-128 (Veröffentlichungen zur bayerischen Geschichte und Kultur; 18); Julia Wood Kramer: This, Too, Is For the Best. Simon Kraemer and his stories. New York: P. Lang 1989.

7 Florian Krobb: Selbstdarstellungen. Untersuchungen zur deutsch-jüdische Erzählliteratur im neunzehnten Jahrhundert. Würzburg: Königshausen \& Neumann 2000, S. 21.

8 [Abraham Kohn]: Die Nothwendigkeit religiöser Volks- und Jugendschriften. In: Wissenschaftliche Zeitschrift für jüdische Theologie 4 (1839), S. 26-36, hier: S. 26. 
brochene Traditionskette und Begeisterung für das Eigene sollten die Leser erfüllt werden, zumal sich im Schoße der christlichen Mehrheitsgesellschaft sich diese nicht von selbst einstellten: »weit und breit zerstreut « lebten die Israeliten, »als kleine Gemeinden oder einzelne Familien«, argumentiert der Hohenemser Rabbiner. ${ }^{9}$ Nicht biblische (oder anderweitig mythologisch etablierte) Helden taugten zur Identifikationsfigur, sondern »tüchtige[] und ehrliche[] jüdische[] Handwerks- und Ackerleute ${ }^{10}{ }^{10}$ "mittlere Helden« also, deren Fähigkeiten und Leistungen sie zu Großem befähigten. Manifestierte sich im Verlangen nach deutschsprachiger Literatur bereits die Überwindung einer alten Ordnung, musste diesem auch in der Wahl von Stoff, Gattung und Präsentationsweise Rechnung getragen werden. Im Gegensatz zu Philippson schwebte Kohn und Krämer kein historischer Heroismus vor, sondern ein zeitgenössischer, unmittelbar exemplarischer, wie ihn Letzterer in seinen Jugendschriften auch tatsächlich umsetzte.

Die neue Volksliteratur in Landessprache hielt sich nicht ausschließlich an die Vorgaben der zeitgenössischen Unterhaltungs- und Trivialliteratur, sondern fühlte sich durchaus der kanonischen Literatur verpflichtet. ${ }^{11}$ In Form von Intertexten wurde ein Bezug zu den Werken der deutschen Klassik, oftmals dem bürgerlichen Trauerspiel, hergestellt, die sich selbst ebenso wenig zur uneingeschränkten Nachahmung eignete wie die Trivialliteratur, und das aus begreiflichen Gründen: Die Probleme der Zeit in ihrer Komplexität und oftmaligen Ausweglosigkeit darzustellen oder gar zu ironisieren, konnte und durfte die jüdische Populärliteratur nicht, wollte sie ihre eigenen Ideale nicht verraten. Dazu zählte allen voran der Glaube an die Integrität der Institution Familie, zu dessen Inbegriff die jüdische in ihrem engen Zusammenhalt und ihrer trauten Heimeligkeit stilisiert wurde. Denn das in den oft sentimentalen Schriften vertretene Bild des Judentums war ein durch und durch bürgerliches, genauer gesagt eines, das die Werte der bürgerlichen Kultur und jüdische Tradition miteinander in Einklang

9 Abraham Kohn, geb. 1807 im böhmischen Zalužany und gest. 1848 in Lemberg, war Reformrabbiner und ab 1833 als Rabbiner in Hohenems tätig, bevor er 1844 eine Predigerstelle in Lemberg annahm. Zum Bezirksrabbiner ernannt, setzte er sich vehement für die Verbreitung des Reformjudentums in Galizien ein, stieß dabei aber auf erheblichen Widerstand. 1848 starb er an den Folgen einer Lebensmittelvergiftung, wobei der Verdacht der vorsätzlichen Vergiftung durch seine orthodoxen Widerstreiter durchaus begründet scheint (Moshe Landau: Kohn, Abraham. In: Encyclopaedia Judaica, Bd 10, Sp. 1143).

10 [Kohn,] Die Nothwendigkeit religiöser Volks- und Jugendschriften (wie Anm. 8), S. 32.

11 S. dazu Jonathan Hess: Middlebrow Literature and the Making of German-Jewish Identity. Stanford: Stanford University Press 2010 (Stanford Studies in Jewish History and Culture), S. 65. 
bringen sollte. Wollte man sich in die aufstrebende (gebildete) Mittelschicht integrieren, musste man auch die religiösen Formen und Inhalte dementsprechend neu bestimmen und berief sich auf die häusliche Sphäre als primären Schauplatz religiöser Übung:

One of the most prominent means by which Jews sought to enter into the ranks of the middle classes was by revitalizing and repacking their religious rituals and traditions, by creating what historians such as Marion Kaplan, Paula Hyman, and Benjamin Baader have described as distinctly bourgeois forms of Judaism focused on home, family life, and the role of women inside and outside the domestic sphere. In nineteenth-century Jewish life, traditionally male realms such as Talmud study lost ground, giving way to public expressions of Judaism - prayer and study in the vernacular, the elevation of women's natural propensity for piety as a general model, or the phenomenon of the rabbi as a novelist, for that matter that in earlier generations would have been seen as female and as inappropriate for Jewish men. ${ }^{12}$

Die Inszenierung und Exemplifizierung einer bürgerlichen Frömmigkeit aus der Fundgrube jüdischer Tradition und Geschichte war genau das, was der neuen Volksliteratur zu ihrer Beliebtheit und Langlebigkeit verhalf: »[...] it was the middlebrow nature of this literature that was the key to its success. German-Jewish belles lettres excelled when it came to combining disparaged traditions of sensationalist melodrama with claims to represent the epitome of high culture. ${ }^{13} \mathrm{Nicht}$ nur ihres eigenen Judentums sollten die Leser versichert werden, sondern ihres legitimen Anspruchs auf die deutsche Kultur, an der zu partizipieren man als sittliche Pflicht verstand: »[...] this middlebrow fiction was clearly meant to supplant the high cultural giants it introduced its readers to indirectly. German-Jewish belles lettres was designed to be consumed alongside other, less markedly Jewish literature produced by non-Jews and Jews alike. ${ }^{14}$ Jonathan Hess, der die Wirkmacht dieser konfessionell-jüdischen Erzählliteratur sehr hoch einschätzt, sieht diese auch maßgeblich an der Bildung eines neuen Ethos beteiligt, für das die intellektuelle Auseinandersetzung mit jüdischer Geschichte und jüdischen Belangen zum gemeinschaftsstiftenden Moment und quasi Religionssurrogat wird: »[...] popular literature [...] helped create a world in which Jewish identity could become a function of reading secular literature. $\aleph^{15}$ Oder wie Florian Krobb formuliert: »[...] gerade die ১Poesieく [der jüdischen Religionspraxis, A. L.] (im Gegensatz $\mathrm{zu}$ theologischer »Wahrheit«) wie der soziale Zusammenhalt der jüdischen

\footnotetext{
12 Ebd., S. 20f.

13 Ebd., S. 19.

14 Ebd., S. 19.

15 Ebd., S. 14.
} 
Schicksalsgemeinschaft (im Gegensatz zu Glaubensgemeinschaft) konnten ja als Elemente der Identität auch eines säkularisierten, verbürgerlichten Judentums gelten. ${ }^{16}$ Die Integration in die deutsche Kultur des Bürgertums sollte, und das sei festgehalten, aus dem Bewusstsein einer jüdischen Sonderstellung, als stolzer »Israelit« erfolgen, wofür Skolnik den von Rosenzweig geprägten Begriff der »Dissimilation « vorschlägt. ${ }^{17}$

Selbstverständlich gestaltete sich die deutsch-jüdische Erzählliteratur im 19. Jahrhundert nicht einheitlich, sondern bildet ein Korpus von Texten unterschiedlicher Qualität und Ausrichtung. Je nach Autor und Werk tendieren manche Schriften (oftmals Jugendbücher) eher zur Trivialisierung, während andere weniger offensichtlich und zielstrebig ihre Absichten verfolgen und durchaus ansprechend gestaltet sind. Shedletzky hält fest, »dass die offizielle jüdische Belletristik in Deutschland, in Theorie und Praxis, im Rahmen der religiösen Reformbewegung ihren Anfang hatte, als Mittel zur Verbreitung ihrer Ideen. Wie in der Entwicklung der religiösen Wochenschriften, folgte auch hier nach der Initiative der Reformbewegung die orthodoxe Reaktion «. ${ }^{18}$ Dies geschah in den 1860er Jahren, als etwa Sara Hirsch und Markus Lehmann ihre schriftstellerische Tätigkeit aufnahmen und ihre Erzählungen in den Zeitschriften und Periodika der Neoorthodoxie wie Jeschurun (1855-1887) und Der Israelit (1860-1938) oder als selbständige Publikationen veröffentlichten.

Als die »drei vorherrschenden Erzählgattungen der deutsch-jüdischen Literatur im 19. Jahrhundert « gibt die einschlägige Literatur den historischen Roman, die Ghettogeschichte und Erzählungen aus der Gegenwart bzw. den Zeitroman an. ${ }^{19}$ Beherrschten anfangs heroisch-historische Erzählungen die literarischen Beilagen und frühen Schriftenreihen, entwickelte sich ab Mitte des Jahrhunderts »der Genre der Ghettogeschichte so stark, dass er in den allgemeinen und jüdischen Literaturgeschichten als Prototyp der deutsch-jüdischen Belletristik des 19. Jahrhunderts erscheint. ${ }^{20}$ Gegen Ende des Jahrhunderts setzten sich dann der »Zeitroman und die Zeitnovelle als dominante Gattungen ${ }^{21}$ durch.

16 Krobb, Selbstdarstellungen (wie Anm. 7), S. 42.

17 Jonathan Skolnik: Jewish Pasts, German Fictions. History, Memory, and Minority Culture in Germany, 1824-1955. Stanford: Stanford University Press 2014 (Stanford Studies in Jewish History and Culture), S. 2.

18 Itta Shedletzky: Literaturdiskussion und Belletristik in den jüdischen Zeitschriften in Deutschland 1837-1918. Jerusalem (Diss.) 1986, S. 97.

19 Krobb, Selbstdarstellungen (wie Anm. 7), S. 12.

20 Shedletzky, Literaturdiskussion und Belletristik (wie Anm. 18), S. 116.

21 Ebd., S. 116. 
Die Entstehung einer säkularisierten jüdischen Erzählliteratur aus dem Bedürfnis heraus, den inneren Zusammenhalt und Verbleib des Individuums im Gemeindeverband zu fördern sowie identitätsstiftende Impulse in Zeiten des Umbruchs zu geben, ist kein ausschließlich deutsches Phänomen. In Frankreich hatte, wie Samuels in seiner einflussreichen Monographie vermerkt, eine ganz ähnliche Entwicklung bereits mehrere Jahre zuvor eingesetzt. Gelten die französisch-jüdische und deutsch-jüdische Literatur gemeinhin als Vorreiter auf diesem Gebiet, zeigt sich die jiddische Novellistik in ihren Ansätzen ebenfalls früh, gelangt allerdings erst ab den 1860ern mit dem Erscheinen von Feuilletongeschichten zur Entfaltung und mit dem Werk S. J. Abramowitsch' (Pseud. Mendele Moicher-Sforim) zum Durchbruch. Etwa zeitgleich entdeckte die sephardische Presse des Osmanischen Reiches die Fortsetzungsgeschichte auf Ladino. Eine der französischen und deutschen vergleichbare englischsprachige Literatur entwickelte sich vor allem in Amerika ebenfalls erst in der zweiten Hälfte des 19. Jahrhunderts. ${ }^{22}$

Den bedeutenden Stellenwert dieser bewusst volksnahen Literatur für die Formierung einer jüdischen Mittelschicht im 19. Jahrhundert erkannt und auf den Punkt gebracht zu haben, ist ein Verdienst der Forschung der letzten Jahre und Jahrzehnte. Genannt seien hier die maßgebenden Studien von Florian Krobb (Selbstdarstellungen, 2000) und Jonathan Hess (Middlebrow Literature and the Making of German-Jewish Identity, 2010) sowie Jonathan Skolniks eingehende Analysen des deutsch-jüdischen historischen Romans (Jewish Pasts, German Fictions, 2014). Nicht unerwähnt sollen in diesem Zusammenhang die äußerst verdienstvollen Arbeiten von Itta Shedletzky, Hans Otto Horch und Gabriele von Glasenapp zur jüdischen Erzähl- und »Ghettoliteratur « bleiben, in denen ein großer Teil des populären Schrifttums des 19. Jahrhunderts erstmals systematisch erfasst wurde. ${ }^{23}$

22 Alle Ausführungen nach Maurice Samuels: Inventing the Israelite. Jewish Fiction in Nineteenth-Century France. Stanford: Stanford University Press 2010 (Stanford Studies in Jewish History and Culture), S. 26f.; Hess, Middlebrow Literature and the Making of German-Jewish Identity (wie Anm. 11), S. 9.

23 Shedletzky, Literaturdiskussion und Belletristik (wie Anm. 18). - Horch, Auf der Suche nach der jüdischen Erzählliteratur (wie Anm. 2). - Gabriele von Glasenapp: Aus der Judengasse. Zur Entstehung und Ausprägung deutschsprachiger Ghettoliteratur im 19. Jahrhundert. Tübingen: Niemeyer 1996 (Conditio Judaica. Studien und Quellen zur deutsch-jüdischen Literatur- und Kulturgeschichte; 11). - Gabriele von Glasenapp/Hans Otto Horch: Ghettoliteratur. Eine Dokumentation zur deutsch-jüdischen Literaturgeschichte des 19. und frühen 20. Jahrhunderts. Tübingen: Niemeyer 2005 (Conditio Judaica. Studien und Quellen zur deutsch-jüdischen Literatur- und Kulturgeschichte; 53-55). 
In den folgenden Kapiteln wollen wir uns, wenn auch nicht ausschließlich, doch weitgehend mit dieser Literatur befassen - der volksnahen deutsch-jüdischen Erzählliteratur: von jüdischen Autoren, zu jüdischen Themen und vorwiegend für eine jüdische Leserschaft; nicht für die intellektuellen Eliten, sondern für den Stand, der im 19. Jahrhundert zunehmend an Gestalt und Einfluss gewinnt: die bürgerliche Mittelklasse. 